\title{
Le destin politique de la mémoire du nationalisme camerounais : entre réhabilitation et rejet
}

Au cours d'une visite officielle du président français François HOLLANDE en terre camerounaise en juillet 2015, une déclaration de ce dernier aura particulièrement marqué l'opinion publique camerounaise et retenu l'attention des commentaires. En effet, le président français déclarait : "C'est vrai qu'il y a eu des épi-

L'auteur : Pierre Авомо est doctorant en Sociologie politique à l'Institut d'étude du développement économique et social (IEDES), Université Paris 1 Panthéon-Sorbonne. sodes extrêmement tourmentés, et tragiques même. Puisqu'après l'indépendance, il y a eu une répression en Sanaga maritime, au pays Bamiléké et nous sommes, comme je l'ai fait partout, ouverts pour que les livres d'histoire puissent être ouverts; et les archives aussi »'. Par cette déclaration, il levait ainsi un tabou et reconnaissait implicitement le rôle de la France dans la répression sanglante exercée contre le mouvement nationaliste camerounais et ses leaders. Cependant, il s'était bien gardé de prononcer des noms, de caractériser les «épisodes extrêmement tourmentés, et tragiques " auxquels il faisait référence, ou encore de désigner les responsabilités, notamment en indiquant le rôle joué par le pouvoir colonial de l'époque. Toutefois, cette déclaration, la première du genre de la part du plus haut représentant de l'ex-puissance coloniale, a suffi pour réactualiser le débat et remettre au goût du jour la question de la mémoire des nationalistes camerounais. La question du destin politique de la mémoire nationaliste camerounaise pèse d'un poids considérable dans les relations franco-camerounaises, et structure à la fois l'histoire et le jeu politiques du Cameroun contemporain. Et pourtant, son évocation ou sa présence dans le débat public au Cameroun est longtemps resté - et reste encore dans une large mesure - un chemin pavé d'embûches aussi bien pour les acteurs politiques que pour le chercheur.

\footnotetext{
'Voy. RFI, 2015, "Au Cameroun, François Hollande brise un tabou », disponible à l'adresse suivante http:// www.rfi.fr/, 3 juillet 2015, (consultée le 26 juin 2016) ; voy. également SHEMA Eugène. C., , "Les questions abordées durant la visite d'État de François Hollande ", Le journal du Cameroun, 5 juillet 2015, disponible à l'adresse suivante : journalducameroun.com, (consultée le 26 juin 2016); "S.E Paul Biya et François Hollande face à la Presse au Palais de L'unité ", Vidéo de la conférence de presse entre les présidents camerounais et français, Youtube, 2015, disponible à l'adresse suivante https://www.youtube.com/, (consultée le 26 juin 2016).
} 
L'une des embûches consiste à définir ce que l'on entend par "nationalistes camerounais ". En effet, que recouvre ce vocable et qui en sont les destinataires? Face à cette question, plusieurs grilles de lecture existent. D'abord, on accole historiquement ce vocable de "nationalistes camerounais " aux militants du premier parti politique camerounais, à savoir l'Union des Populations du Cameroun (UPC) ${ }^{2}$, et plus particulièrement à ses leaders successifs morts sous le coup de la répression ante et post coloniale. Ensuite, une deuxième grille de lecture, thèse officielle gouvernement camerounais, tend à considérer que cette dénomination n'est pas l'exclusive des militants upécistes morts pendant et après l'indépendance. Les défenseurs de cette vision font une distinction entre les « nationalistes radicaux » ou " extrémistes » et les «nationalistes modérés » que seraient les membres des autres mouvements politiques des années d'indépendance militant pour la continuation du lien avec l'ex-puissance coloniale. Mais cette thèse du nationalisme modéré et extrémiste est battue en brèche par Abel EYINGA et d'autres ${ }^{3}$. Enfin, une autre acception du nationalisme camerounais, non conflictuelle avec la première, élargit le spectre des nationalistes camerounais pour y inclure les résistants à la pénétration allemande au Cameroun ${ }^{4}$. En tout état de cause, l'appellation de "nationaliste camerounais " charrie beaucoup de convoitises et constitue un enjeu symbolique et politique indéniable.

Au regard de ces controverses, il convient d'apporter des clarifications sur le concept de nationalisme et comment il sera appréhendé dans cette étude relativement au cas camerounais. D'après Louis BALTHAZAR, ce concept désigne " un mouvement qui consiste à accorder une priorité à l'appartenance nationale et à lutter pour une meilleure reconnaissance de la nation à laquelle on appartient $\|^{5}$.

\footnotetext{
${ }^{2}$ Voy. à ce propos les perspectives d'analyse respectives de DOMERGUE Manuel, TATSITSA Jacob et DELTOMBE Thomas, Kamerun! Une guerre cachée aux origines de la Françafrique, Paris, La Découverte, 2011,744 p. ; Eyinga Abel, L'U.P.C: Une révolution manquée?, Paris, Africa Chaka, 2004, 196 p. ; JosePH Richard, Le Mouvement nationaliste au Cameroun: Les Origines sociales de l'UPC, Paris, Karthala, 2000,414 p. ; Manga Olomo Jocelyn et Ntuda Ebode Jean Vincent, Divisions au Coeur de l'UPC Contribution à la Connaissance de l'Histoire Politique du Cameroun, Paris, Editions L'Harmattan, 2011, 204 p. ; MBEMBE Achille, La naissance du maquis dans le Sud-Cameroun, Karthala, 1996, 430 p.; MBEMBE Achille, "La palabre de l'indépendance : les ordres du discours nationaliste au Cameroun (1948-1958) ", Revue française de science politique, 35, 3, 1985, pp. 459-487.

3 Voy. EYInGA Abel, L'U.P.C: Une révolution manquée?, op. cit.; JosEPH Richard, Le Mouvement nationaliste au Cameroun : Les Origines sociales de l'UPC, op. cit.; MBEMBE Achille, "Pouvoir des morts et langage des vivants : Les errances de la mémoire nationaliste au Cameroun », op. cit., p. 38.

4 Voy. SEGNOU Etienne, Le nationalisme camerounais dans les programmes et manuels d'histoire, Editions L'Harmattan, 2015, $392 \mathrm{p}$.

${ }^{5}$ Balthazar Louis, Bilan du nationalisme au Québec. Montréal : Éditions de L'Hexagone, Collection : Politique et société, 1986, p. 13.
} 
D'après cette définition, on peut dégager trois critères permettant de caractériser le fait nationaliste : Il faut d'abord la présence d'un mouvement, ou groupe organisé ; ensuite la priorité accordée à l'appartenance nationale qui peut se matérialiser d'une part, par la prééminence des intérêts de la nation (existante ou en formation) sur les intérêts particuliers; et d'autre part par la préférence accordée aux intérêts nationaux par rapport aux intérêts étrangers. Enfin, la volonté du groupe de lutter pour voir triompher la cause nationale défendue, cette lutte pouvant aller jusqu'à l'affrontement armé sous la forme des guerres de libération nationale, notamment dans les contextes d'occupation étrangère ou de décolonisation.

Ainsi, à la lumière de ce qui précède, le concept de nationalistes camerounais sera employé pour désigner les leaders upécistes dans la mesure où ces derniers, à travers leur action, cumulent les trois critères du nationalisme découlant de la définition de Louis BALTHAZAR ci-dessus proposée. Bien que l'on ne puisse pas nier dans l'absolu la volonté d'accorder une préférence aux intérêts camerounais ou la revendication de l'indépendance à d'autres groupes politiques camerounais de l'époque, la particularité de l'UPC reposait au moins sur trois données historiques: Premièrement, l'UPC et ses leaders étaient les premiers et seuls acteurs revendiquant l'indépendance et la réunification du Cameroun, et qui furent contraints d'engager une lutte armée contre le pouvoir colonial relayée par la suite par leurs alliés locaux. Deuxièmement, en se référant aux propos des autorités françaises, notamment la déclaration ci-dessus faite par le président français, à la "Sanaga maritime " et " au pays Bamiléké », il apparaît clairement qu'il fait référence à l'UPC. Troisièmement, «l'UPC fut le tout premier parti politique à revendiquer l'indépendance du Cameroun face à l'oppression de l'occupant. De ce fait, il a marqué l'histoire du Cameroun comme aucune autre organisation ${ }^{6}$. II serait donc historiquement incongru de ranger dans la même catégorie des groupes ayant combattu, les uns pour l'indépendance, et donc clairement nationalistes et anticolonialistes, et les autres contre l'indépendance, et donc ostensiblement anti-indépendantistes et pro-coloniaux. ${ }^{7}$

Cette clarification conceptuelle et historique opérée n'épuise cependant pas la question des tergiversations et frilosités autour de la réhabilitation de la mémoire des nationalistes au Cameroun. Le souve-

\footnotetext{
- Manga Olomo Jocelyn et Ntuda Ebode Jean Vincent, Divisions au Coeur de l'UPC Contribution à la Connaissance de l'Histoire Politique du Cameroun, Paris, Editions L'Harmattan, 2011, p. 14.

${ }^{7}$ Voy. EYINGA Achille, L'U.P.C: Une révolution manquée?, op. cit.; JoSEPH Richard, Le Mouvement nationaliste au Cameroun: Les Origines sociales de l'UPC, op. cit.; MBEMBE Achille, "Pouvoir des morts et langage des vivants : Les errances de la mémoire nationaliste au Cameroun », op. cit.
} 
nir de ces acteurs ayant combattu pour l'indépendance du Cameroun est sinon en voie d'être oublié, du moins discrètement entretenu et pas du tout assumé publiquement. Et lorsqu'on en vient à l'évoquer publiquement, c'est bien souvent dans une approche confuse et contradictoire avec les récits historiques. II s'agit donc ici d'expliquer comment, en dépit de la reconnaissance historique du fait nationaliste au Cameroun, la mémoire nationaliste y reste une question relativement occultée dans l'espace politique national. Dans cette étude, trois hypothèses sont proposées pour tenter d'expliquer ce rapport particulier qu'entretient le Cameroun avec sa mémoire nationaliste. Dans un premier temps, cette occultation de la mémoire nationaliste pourrait s'expliquer par les pesanteurs qu'elle a dans l'histoire de la relation franco-camerounaise qui a été marquée par un processus de décolonisation teinté d'un conflit resté latent et toujours structurant du comportement des acteurs politiques aussi bien camerounais que français. Dans un second temps, le rejet de la mémoire des nationalistes camerounais pourrait s'expliquer par la menace de l'épée de Damoclès que fait planer l'éventualité d'une réhabilitation des nationalistes susceptible de dégénérer en un conflit de légitimités: d'une part, la légitimité historique et mémorielle qui incombe aux leaders nationalistes défaits par la puissance coloniale et ses successeurs; et d'autre part, la légitimité formelle des " héritiers " du pouvoir colonial. Cette menace que fait peser le poids du "pouvoir des morts " ${ }^{8}$ justifiera par la suite un révisionnisme et une instrumentalisation institutionnels et politiques de tous les côtés. Troisièmement, la question de la réhabilitation de la mémoire nationaliste dans l'espace politique camerounais se heurte également à la confusion orchestrée et entretenue par les acteurs politiques du pouvoir et de l'opposition.

Sous ce rapport, le destin politique de la mémoire des nationalistes camerounais sera examiné d'abord comme la réminiscence d'un contentieux de la décolonisation non épuisé sur le fond (1); ensuite comme l'expression de la crainte d'un conflit des légitimités a priori défavorable au pouvoir ante et post indépendance (2); et enfin, comme la conséquence des confusions orchestrées et entretenues par le pouvoir et les « héritiers » de l'opposition (3).

\footnotetext{
${ }^{8}$ MBEmBE Achille, "Pouvoir des morts et langage des vivants : Les errances de la mémoire nationaliste au 


\section{La réminiscence d’un contentieux de la décolonisation non épuisé sur le iond}

La première hypothèse permettant d'expliquer les atermoiements autour de la mémoire nationaliste camerounaise réside dans le tabou créé autour de celle-ci. Ce tabou s'explique en partie par la réminiscence d'un contentieux de décolonisation mal géré dont les conséquences matérielles et symboliques continuent à hanter les esprits. Pour mieux rendre compte de cette réminiscence, il convient de revenir sur la guerre d'indépendance ayant opposé les forces coloniales et post coloniales aux nationalistes camerounais, d'analyser la défaite de ces derniers et de souligner la poursuite du mouvement de répression post-indépendance.

\section{Contours et circonstances d'une guerre imposée entre la puissance coloniale et le mouvement nationaliste}

Dans la majeure partie des ex-colonies françaises d'Afrique noire, le processus d'indépendance avait été négocié9 ou concédé sans affrontement entre l'ex-puissance coloniale et les élites locales. Cependant, au Cameroun comme en Algérie avec certes des résultats différents, le processus d'indépendance a été précédé, voire précipité, par une sanglante guerre d'indépendance ${ }^{10}$.

En Algérie, profitant de la défaite de l'armée française en Indochine, les indépendantistes algériens vont créer au Caire en 1954, le «Front de Libération Nationale " (FLN) pour revendiquer l'indépendance". Face aux fins de non-recevoir présentées par la puissance coloniale, ils vont d'abord se lancer dans des actions insurrectionnelles de contestation de la présence coloniale française, et par la suite engager une guerre asymétrique contre les forces françaises. Bénéficiant d'une meilleure organisation politique et militaire, d'une plus grande cohésion idéologique, les indépendantistes algériens, contrairement à leurs homologues camerounais de l'UPC'12, parviendront à obtenir

\footnotetext{
"Voy. Droz Bernard, "Regards sur la décolonisation de l'Afrique Noire », Labyrinthe, 16, 2003, p. 918 ; PATHÉ Anne-Marie, "La France et l'indépendance de l'Afrique noire ", Vingtième Siècle. Revue d'histoire, 1991, n³0, p. 108.

10 Voy. EyInga Abel, L'U.P.C: Une révolution manquée?, op. cit.; JosEPH Richard, Le Mouvement nationaliste au Cameroun : Les Origines sociales de l'UPC, op. cit.; MBEMBE Achille, "Pouvoir des morts et langage des vivants : Les errances de la mémoire nationaliste au Cameroun », op. cit.

"Chaulet Achour Christiane, "Guerre de libération nationale/Guerre d'Algérie (1954-1962) ", in La France et l'Algérie en 1962, Editions Karthala, 2013, p. 307. 
l'indépendance de l'Algérie. En effet, ne bénéficiant pas des mêmes circonstances que le FLN, notamment l'effet de surprise des évènements de la "Toussaint rouge $»^{13}$ qui ont peu alerté les autorités françaises de la métropole, les nationalistes de l'UPC vont affronter une puissance coloniale française murie par deux expériences de défaites successives en Indochine, puis en Algérie. Ces deux expériences ont permis à la France de développer une stratégie militaire contre-insurrectionnelle qui fera ses preuves face aux Upécistes mal outillés du Cameroun. Pour la France, la bataille contre l'UPC était une question d'honneur militaire à restaurer. C'est sans doute l'une des raisons pour lesquelles la répression du mouvement indépendantiste camerounaise fut l'une des plus extrêmes de la décolonisation française. ${ }^{14}$

Cette guerre de la France contre l'UPC est en réalité considérée par plusieurs observateurs comme un conflit ayant été imposé aux forces nationalistes et indépendantistes. En effet, «le mouvement nationaliste camerounais fut en butte à une provocation délibérée du gouverneur Roland PRÉ, qu'il ne sut déjouer $\aleph^{15}$. Cette affirmation est d'ailleurs étayée par le témoignage d'un des nationalistes camerounais Ndeh NTUMAZAH ${ }^{16}$, ancien dirigeant du mouvement indépendantiste UPC :

L'UPC n'a jamais pensé qu'un jour elle allait être obligée de prendre les armes contre le colonialisme au Kamerun. Le seul crime que les Français ont reproché à l'UPC est la proclamation de Douala en 1955. Cette proclamation qui faisait pression sur la France et les Nations Unies visait à accélérer le processus d'indépendance du Kamerun. C'est après cette proclamation que les Français ont engagé une lutte féroce contre tous les Upécistes du Kamerun. Mais nous tenions à préserver l'étoile de l'UPC. Pour cette raison, nous avons décidé de quit-

\footnotetext{
${ }^{13}$ BAT Jean-Pierre, Récits d'historien - La Toussaint rouge, Hatier, 2014, 192 p.

${ }^{14}$ Domergue Manuel, TATSITSA Jacob et Deltombe Thomas, Kamerun! Une guerre cachée aux origines de la Françafrique, op. cit.

${ }^{15}$ BAYART Jean-François, «L'U.P.C., le gouvernement Ahidjo », Le Monde diplomatique, 1 janvier 1979; JOSEPH Richard, Le Mouvement nationaliste au Cameroun : Les Origines sociales de l'UPC, op. cit.

${ }^{16}$ Wilson NDEH NTUMAZAH, homme politique engagé, un des premiers militants anticolonialiste camerounais, figure historique de l'histoire de la libération et de l'indépendance du Cameroun, décède à Londres le 21 janvier 2010 à l'âge de 84 ans. En 1950, il marque son entrée en politique par son adhésion à l'UPC (I'Union des Populations du Cameroun) où il va retrouver Ernest OUANDIÉ et UM NYOBÈ, entre autres. En 1955, le parti est interdit d'exercer ses activités sur le territoire camerounais. Ndeh NTUMAZAH crée alors un autre parti baptisé "One Kamerun Movement», en abrégé "Ok», dont-il assure la présidence. Son parti va se positionner dans le même esprit de combat dit " pour la libération du Cameroun " que l'UPC. Voy http://prismacanal. blogs.nouvelobs.com, (consultée le 28 mai 2016).
} 
ter la scène politique. Um NYoBÉ s'est alors retranché dans la Sanaga Maritime... Il s'agissait maintenant pour nous de définir une stratégie pour faire face aux colons. Je dois dire ici que l'oppression donne naissance à la résistance ${ }^{17}$.

Ainsi, il apparait que malgré l'apparence du discours et l'air du temps à cette époque ${ }^{18}$, l'intention des autorités coloniales françaises n'était pas de décoloniser, ou en tout cas de le faire de façon immédiate avec de vrais interlocuteurs. Après sa création, I'UPC s'était rapidement imposée comme la première force politique camerounaise pouvant légitiment prétendre succéder au pouvoir colonial. Ainsi, dès 1948, l'UPC voit son affiliation au Rassemblement Démocratique Africain ${ }^{19}$ (RDA) confirmée et Um NYOBÈ son leader est porté à la vice-présidence de ce mouvement panafricain. Le 4 octobre 1952, la direction de l'UPC adresse écrit à l'ONU pour demander l'envoi de pétitionnaires. Cette demande est acceptée, et Um NYOBÈ est choisi par ses camarades de l'UPC pour se rendre à New York et y prendre la parole au nom du Cameroun. Cette audience internationale accordée à l'UPC suscitera la protestation des autorités coloniales, par la voix du Dr Aujoulat, président de l'Assemblée Territoriale du Cameroun (ATCAM). Aussi, lors de la deuxième audition à laquelle l'UPC sera invitée à prendre la parole, le pouvoir colonial dépêchera deux personnes pour lui porter la contradiction, en l'occurrence Charles OKALA ${ }^{20}$ et Alexandre Douala MANGA BELL21. De même, lors des interventions suivantes à l'ONU, ce sera au tour de Guillaume BISSECK ${ }^{22}$, puis de Daniel KÉMAJOU ${ }^{23}$ de porter la contradiction à l'UPC.

\footnotetext{
${ }^{17}$ BIAmOU Théophile, "Wilson Ndeh Ntumazah parle (Entretien) », La Nouvelle Expression, 27 janvier 2010. ${ }^{18}$ Voy. OULMONT Philippe et VAïsse Maurice (Ouvrage dirigé par), De Gaulle et la décolonisation de I Afrique subsaharienne, Paris, Karthala, 2014, $252 \mathrm{p}$.

${ }^{19}$ Le RDA était un mouvement politique à vocation panafricaine, proche du mouvement communiste français créé suite au Congrès de Bamako par Félix HouPHOUËT-BoIGNY et Modibo KEITA. II regroupait les différents partis politiques des principaux États francophones d'Afrique noire, notamment la Côte d'Ivoire, le Gabon, le Cameroun, la Guinée, le Sénégal, le Niger, le Tchad, la Haute-Volta (actuel Burkina Faso), le Soudan français (actuel Mali) et le Congo. Toutefois, en 1950, le RDA change d'orientation idéologique à la suite des répressions ayant entraîné de nombreux morts, notamment en Côte d'Ivoire. II cesse d'être ouvertement anti-colonialiste et se résigne à la collaboration avec l'Administration coloniale.

${ }^{20}$ Ancien sénateur de la IV République française et ancien membre des gouvernements camerounais sous Ahmadou AHIDJO.

${ }^{21}$ Personnage issu d'une longue lignée aristocratique du peuple Sawa du Cameroun, c'est un Ancien député sous la IVe République française.

${ }^{22}$ Leader de l'ESOCAM (Evolution Sociale Camerounaise), parti créé en 1949 juste après l'UPC pour s'opposition à la vision de réunification et d'indépendance du Cameroun défendue par I'UPC. II bénéficiait d'un soutien fort de l'Administration coloniale. 
En interne, le pouvoir colonial va encourager et pousser à la création de mouvements politiques anti-indépendantistes et favorables à sa cause. Ce sera d'abord la création de l'ESOCAM de Guillaume BISSECK, puis du Mouvement de Coordination des Indépendants Camerounais (INDECAM), et enfin la Renaissance Camerounaise (RENAICAM). Tous ces mouvements furent confinés à quelques parties du territoire camerounais sans véritablement enclencher une adhésion comparable à celle de l'UPC. Face à cet échec, les autorités coloniales vont adopter une autre stratégie. Celle-ci va consister à combattre le mouvement nationaliste aux revendications duquel elles étaient hostiles. Pour légitimer cette guerre, une stratégie politique et militaire sera adoptée.

Au plan politique, il s'agissait de diaboliser le mouvement nationaliste en l'accusant d' "insurrection", de "rébellion", de "terrorisme " ou d'être responsable de "désordres sanglants " ${ }^{24}$. "La Presse du Cameroun parle de terroristes, bandes terroristes, commando terroriste, criminels, bandits, hors-la-loi, transfuges. Le journal catholique, l'Effort Camerounais, dénonce ces bandes criminelles qui sèment la mort et la désolation ${ }^{25}$.

Le deuxième élément de cette stratégie de diabolisation consistait à taxer l'UPC de mouvement communiste, affilié au mouvement communiste français et dépendant de lui :

\begin{abstract}
Le communisme, en agitant habilement le drapeau d'un nationalisme naissant et sous son couvert, a pris racine au Territoire et particulièrement en pays Bassa, terrain fertile par excellence. L'hymne camerounais rabâché à longueur de réunions est devenu un chant de ralliement upéciste, et n'est pas, la propagande aidant, sans effet sur les masses et particulièrement les jeunes. L'UPC tout comme le Viet-Minh - et c'est là un des points communs qui révèlent l'influence des théories communistes 'démarquées' pour être adaptées aux populations noires comme elles ont été pour les jaunes - a centré son action sur le nationalisme antiblanc et l'anticolonialisme en évitant toutefois de heurter de front les sentiments traditionalistes du pays ${ }^{26}$.
\end{abstract}

Cette stratégie de diabolisation de l'UPC va donc préparer le terrain pour une répression d'abord administrative, puis militaire contre I'UPC et ses leaders indépendantistes.

\footnotetext{
${ }^{24}$ MBEMBE Achille, "Pouvoir des morts et langage des vivants : Les errances de la mémoire nationaliste au Cameroun », op. cit., p. 39.

${ }^{25}$ ONANA MfEge André-Hubert, "L'armée de libération nationale Kamerunaise et sa stratégie 1959-1970 ", op. cit. p. 260.

${ }^{26}$ MвEMBE Achille, "La palabre de l'indépendance : les ordres du discours nationaliste au Cameroun (19481958) ", Revue française de science politique, 35, 3, p. 460, citant une étude du Colonel LACHEROY intitulée La campagne d'Indochine ou une leçon de la guerre révolutionnaire.
} 
Ainsi, tout commence en 1955 avec l'arrivée du gouverneur Roland PrÉ au Cameroun. Ce dernier orchestre tout d'abord des provocations juridico-policières (Affaire GILIS) qui vont contraindre Um NYOBÈ à entrer dans la clandestinité27. Ensuite, des émeutes sont provoquées à Douala suivie d'une répression "sauvage " ordonnée par le gouverneur Roland PRÉ. Plusieurs morts sont décomptés; le siège de l'UPC à New Bell est "saccagé et réduit en cendres " ${ }^{28}$. Le 13 juillet 1955, le gouvernement français prend un décret prononçant la dissolution et l'interdiction de l'UPC. Dès lors, contraint de disparaître du champ politique officiel, I'UPC va entrer dans le «maquis " pour engager une lutte armée contre le pouvoir colonial.

Au plan militaire, c'est la stratégie de guerre contre-révolutionnaire inspirée des expériences indochinoise, vietnamienne et algérienne qui sera appliquée pour briser la résistance des nationalistes camerounais. Face à la résistance opposée par le mouvement nationaliste, les autorités coloniales ne lésineront pas sur les moyens à adopter. Ils utiliseront toutes les techniques de guerre contre-révolutionnaire pour écraser le mouvement indépendantiste et éliminer la figure emblématique de I'UPC, son leader Um NYOBË ${ }^{29}$ :

\begin{abstract}
Aussi, la rébellion est-elle éprouvée par une répression optimale menée successivement par les forces françaises, puis par les forces conjointes franco-camerounaises. Celles-ci appliquent, à quelques exceptions près, les procédés tactiques hérités de la guerre d'Indochine et en cours en Algérie : action psychologique associant la propagande et l'intimidation des populations, isolement, étouffement et destruction des maquis se trouvant en milieux favorables, regroupement et création de zones interdites ${ }^{30}$.
\end{abstract}

\title{
La défaite des forces nationalistes et l'accession du cameroun à l'indépendance
}

La répression impitoyable conduite par les forces coloniales aboutira à la défaite du mouvement nationaliste. Faiblement équipé, bénéficiant de faibles soutiens internationaux et en Afrique ${ }^{31}$, la défaite des upécistes sera fatale. De plus, le mouvement nationaliste sera coupé

\footnotetext{
${ }^{27}$ Voy. le récit chronologique fait par EYINGA Achille in L'U.P.C: Une révolution manquée?, op. cit. pp. 181-189. ${ }^{28}$ lbid.

${ }^{29}$ Voy. le récit et les témoignages détaillés donnés par DoMERGUE Manuel, TATSITSA Jacob., DeLTOMBE Thomas, in Kamerun! Une guerre cachée aux origines de la Françafrique, Paris, La Découverte, 744 p. 30 OnANA MfEge André-Hubert, "L'armée de libération nationale Kamerunaise et sa stratégie 1959-1970 ", op. cit., p. 263.

${ }^{31}$ Voy. l'analyse D'EssomBA Apollinaire, Le mouvement nationaliste camerounais dans le système international, Paris, Editions L'Harmattan, 2016, 308 p.
} 
de ses soutiens populaires grâce à la campagne d'intimidation et de pression exercée contre la population. Citant La Presse du Cameroun, MFEGE reprend les propos des autorités post-coloniales menaçant de représailles toute personne venant en aide aux upécistes : "L'ordre que nous avons donné, l'ordre que je donne aux autorités militaires et administratives est de traiter ceux qui aident les terroristes exactement comme les terroristes, c'est-à-dire qu'ils doivent être châtiés comme des terroristes ${ }^{32}$.

Ainsi, après la mort de Um NYOBÈ en 1958, puis de Félix Moumié empoisonné au Thallium à Genève en $1960^{33}$, les autres leaders indépendantistes seront successivement éliminés (Ossende AfANA en 1969, Ernest OUANDIÉ en 1971) ou mourront en exil (Abel KINGUÉ en 1964 au Caire $)^{34}$. Le reste de ses cadres dirigeants ayant survécu seront, pour la grande majorité, poussés à l'exil. Une minorité sortie du "maquis» fera alliance avec le pouvoir colonial et ses alliés locaux en abandonnant la lutte armée. Ce sera notamment le cas de Mayi MATIP ou encore d'Emma Oттоu. Défait, mais pas complètement éradiqué, le mouvement nationaliste verra le Cameroun accéder à l'indépendance sans lui en 1960. Ainsi, le pouvoir colonial décidera d'accorder l'indépendance à ceux qui ne l'avaient pas demandée, à ceux qui avaient activement milité contre cette idée et pour une continuation de la relation avec lui. Cette précision s'avère nécessaire pour comprendre l'embarras et la bataille de légitimité historique dans laquelle se lanceront les autorités post-coloniales.

\section{La crainte d'un conilit des légitimités et le bannissement public de la mémoire nationaliste}

La deuxième hypothèse permettant de rendre compte de la frilosité à réhabiliter la mémoire nationaliste camerounaise tient à la crainte d'un conflit des légitimités. De fait, il y a d'une part une légitimité historique, qui de par les circonstances historiques évoqués précédemment, échoie naturellement aux leaders indépendantistes inscrits dans l'histoire de l'indépendance du Cameroun contemporain. D'autre part, il y a ce que l'on pourrait qualifier de légitimité « légale-rationnelle » au sens wébérien qui incombe aux autorités post-coloniales

\footnotetext{
32 OnANA MfEge André-Hubert, "L'armée de libération nationale Kamerunaise et sa stratégie 1959-1970 », op. cit., p. 264.

33 Voy. le récit présenté par LALOuP Laurent, «William Bechtel - Les Français Libres », disponible à l'adresse suivante, http://www.francaislibres.net/ (consultée le 25 mars 2016).

34 OnANA MfEge André-Hubert, "L'armée de libération nationale Kamerunaise et sa stratégie 1959-1970 ", op. cit.
} 
ayant et exerçant l'effectivité du pouvoir dont elles tiennent le fondement dans les instruments juridiques régissant le Cameroun. Ces deux légitimités n'étant point en cohérence, voire s'opposant radicalement, rendent difficile l'objectivation d'un récit national basé sur la mémoire collective. Face à cette incohérence, les autorités post-coloniales vont procéder à ce que MBEMBE qualifie de « refabrication étatique de l'histoire ${ }^{35}$, une tentative de réécriture du récit national camerounais à l'aune des intérêts et des objectifs politiques des autorités post-coloniales.

Ce processus de réécriture du récit national - avec en arrière fond une "dévalorisation de la mémoire » des indépendantistes - se fera à l'aide de deux techniques instrumentales : le bannissement de l'espace public des noms des leaders nationalistes ainsi que la criminalisation de leurs actions et la configuration des programmes éducatifs à l'aune du récit national réécrit.

\section{Le bannissement de la mémoire des nationalistes dans l'espace public}

La première étape du bannissement et de la dévalorisation de l'action des nationalistes consiste à la qualifier de criminelle, menée contre les intérêts des populations. Pour l'ancien président Ahmadou AHIDJO, la période ayant précédé son accession au pouvoir en $1958^{36}$ est celle des "crimes encore présents dans nos mémoires' ", des "'méfaits' ", et "'forfaits' " commis par "ceux qui ont pillé ou tué" ". L'action des nationalistes est qualifiée de "'néfaste et sans issue' ". Par conséquent, il conviendrait d'«'oublier ce passé de violence et de haine' "37 :

\footnotetext{
'Je le dis bien haut : le passé est ce qu'il est. Nous sommes, pour notre part, décidés à regarder vers l'avant (...). Lorsqu'un feu est sur le point de séteindre, il faut se garder d'y jeter des brassées de bois sec'. Du point de vue de M. AHIDJO, I'UPC dont le vocable appartient désormais au passé, a été un mouvement qui a fondé des groupes paramilitaires et utilisé la violence contre ses propres compatriotes, parfois même (...) contre des femmes et des enfants'. Les acteurs politiques
}

\footnotetext{
35 MBEmBE Achille, «Pouvoir des morts et langage des vivants : Les errances de la mémoire nationaliste au Cameroun ", op. cit., pp. 57 et suivantes.

${ }^{36}$ Cette date n'est en effet pas choisie au hasard. Certes, elle constitue bien l'année d'arrivée au pouvoir de M. AHIDJO, mais est surtout celle marquant l'exécution du leader de l'UPC Ruben UM NyoBÈ et le début de la fin du mouvement nationaliste.

37 MBEmbe Achille, «Pouvoir des morts et langage des vivants : Les errances de la mémoire nationaliste au 
qui épousèrent sa cause sont décrits comme des 'frères qui ont été abusés, trompés ou qui ont pu croire par des voies illégales travailler au bonheur du pays'38.

Au plan législatif, l'État va s'appuyer sur l'ordonnance n 62-oF-18 du 12 mars 1962 portant répression de la subversion pour réprimer toute personne évoquant les noms des nationalistes. Cette répression a contribué à installer une peur dans les esprits des camerounais. Cette peur pourrait trouver une explication dans le fait que, «durant une longue période, principalement sous le règne d'Ahmadou AHIDJO, la référence aux nationalistes et à leur lutte était officiellement interdite et passible d'emprisonnement pour subversion "39. Même dans le cadre des recherches scientifiques, cela était interdit. Ce fut par exemple illustré par la difficulté de soutenance du mémoire de DEA d'Achille MBEMBE portant sur La vie quotidienne dans les maquis du Sud-Cameroun de 1955 à 1960, au cours de laquelle tous les membres du jury pressenti se désistèrent. D'après Richard JOSEPH,

\footnotetext{
La peur et l'auto-censure sont tellement omniprésentes dans le Cameroun que le chercheur a conscience en permanence du danger de compromettre quelqu'un en allant simplement l'interroger. A la différence du Kenya où le mouvement des Mau Mau fait partie de l'héritage national et est glorifié comme tel, au Cameroun, ceux qui combattirent et donnèrent leur vie pour l'indépendance ne sont mentionnés que pour être condamnés ${ }^{40}$.
}

Pour asseoir et consolider cette réécriture de l'histoire en marche, l'État va aller plus en profondeur en s'intéressant à l'enseignement de I'histoire.

\section{La config̉uration des prog̉rammes éducatifis à l'aune du récit national réécrit}

Sorti victorieux de la guerre d'indépendance soldée par la défaite des nationalistes, le pouvoir post-colonial va prendre en charge l'enseignement de l'histoire à l'école et à l'université dans une perspective révisionniste en contradiction avec la réalité historique. Aussi, l'histoire va devenir un enjeu politique, particulièrement la période de déco-

\footnotetext{
${ }_{38}$ MBEmbe Achille, "Pouvoir des morts et langage des vivants : Les errances de la mémoire nationaliste au Cameroun », op. cit., p. 58

39 SEGNOU Etienne, Le nationalisme camerounais dans les programmes et manuels d'histoire, Editions L'Harmattan, p. 124.

40 JOSEPH Richard, Le Mouvement nationaliste au Cameroun: Les Origines sociales de l'UPC, op. cit. pp. 22-23.
} 
lonisation. Elle sera enseignée avec le souci de façonner le citoyen nouveau dans la vision idéologique et révisionniste portée par l'Etat post-colonial.

C'est ainsi qu'au primaire, dans le programme officiel d'enseignement daté de 2001, sur les 42 leçons consacrées du programme d'histoire, seulement quatre portent sur le nationalisme camerounais, soit $9,5 \%$ du taux de représentativité. Paradoxalement, la part du programme d'histoire consacrée au reste du monde compte seize leçons, soit $38 \%$ de la part du programme officie| ${ }^{44}$.

Au niveau du secondaire, le même constat paradoxal se dégage aussi bien dans l'enseignement général que dans l'enseignement technique et professionnel. En effet, dans l'enseignement général, le nationalisme camerounais représente trois leçons sur un total de 164, soit $1,8 \%$ du programme officiel, tandis que l'histoire du reste du monde ${ }^{42}$ est fortement représentée à hauteur de $80 \%$ du programme officiel (datant de 1990). Dans l'enseignement secondaire technique et professionnel, sur 66 leçons du programme d'histoire en vigueur en 2001, on en dénombre trois portant sur le nationalisme camerounais, respectivement dans les classes de quatrième, première et terminale, et rien pour les classes de sixième et cinquième.

Enfin, au niveau de l'enseignement supérieur, la tendance est la même, notamment en faculté des lettres et des sciences humaines où

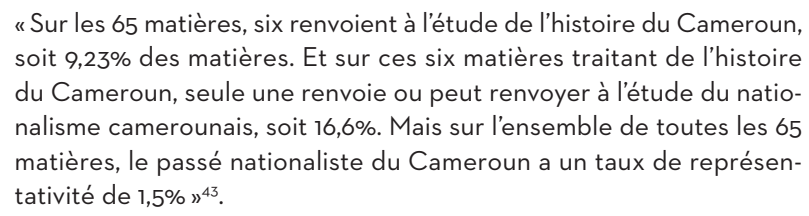

Certes, au niveau primaire, certains noms de nationalistes sont évoqués dans les programmes. On les invoque sous l'intitulé «les résistances à la présence allemande au Cameroun » et celui de "grandes figures de l'histoire du Cameroun de 1956 à 1998 ». "Cependant, il faut aussi noter que l'étude de la résistance des camerounais à la domination étrangère n'est envisagée de manière valorisante que pour celle s'étant manifestée durant la période coloniale allemande. La résistance

\footnotetext{
${ }^{41}$ SEGNOU Etienne, Le nationalisme camerounais dans les programmes et manuels d'histoire, Editions L'Harmattan, p. 114.

${ }^{42}$ Ibid., pp. $115-116$. 
camerounaise ne figure pas explicitement dans les programmes ni même implicitement dans les objectifs des différentes leçons qui s'y rapportent ou qui peuvent s'y rapporter ${ }^{44}$.

En ce qui concerne l'étude des figures de l'histoire de la décolonisation du Cameroun, seul Ruben UM Nrobè est évoqué, alors qu'il existe d'autres leaders de la lutte indépendantiste tels que Félix Moumié, Ossendé Afana, Abel Kingue, Ernest Ouandié, etc. Ainsi, dans une enquête menée auprès des élèves de l'enseignement secondaire général et technique à Douala, il apparaît que sur les douze figures du nationalisme camerounais proposées pour identification visuelle par les élèves, une seule figure se dégage comme connue de tous, en l'occurrence celle du président de l'époque, Ahmadou AHIDJO, suivie de Ruben UM NYOBÈ. Quant aux autres figures nationalistes, ni leur nom, ni leur visage ne sont connus de la majorité des élèves ${ }^{45}$.

De même, d'autres faits révélateurs de cette volonté de bannissement des nationalistes du récit national sont notamment l'absence de mention dans les programmes scolaires de la période de 1955-1971 ou encore l'absence de précision quant aux circonstances, au lieu ou encore moins aux auteurs de l'assassinat de UM NroBè. Il est juste mentionné qu'il a été assassiné. En outre, dans le programme de l'enseignement secondaire,

\footnotetext{
le cours consacré à l'étude des mouvements nationalistes en Afrique ('Les mouvements nationalistes en Afrique') indique de prendre pour exemple quelques mouvements issus des pays d'Afrique centrale francophone, mais le cas du Cameroun avec l'UPC n'est pas mentionné comme exemple à prendre, mais plutôt celui du Tchad ${ }^{46}$.
}

À l'analyse, il se dégage une logique décroissante suivant laquelle plus on progresse dans le parcours scolaire et académique, moins une chance est donnée à l'apprenant de prendre connaissance de et/ou de s'approprier la mémoire nationaliste. Dans un tel contexte, les conditions sont réunies pour, sinon véritablement oublier la portée et le sens du combat nationaliste, du moins créer et entretenir une confusion dans les esprits dommageable à la réhabilitation de la mémoire nationaliste.

\footnotetext{
${ }^{44}$ Ibid., p. 121.

${ }^{45}$ SEGNOU Etienne, Le nationalisme camerounais dans les programmes et manuels d'histoire, p. 122. 


\section{Les coniusions institutionnelles entretenues par les acteurs ou la réhabilitation sans gloire des figurures nationalistes}

Depuis le changement survenu à la tête de l'État en 1982, et plus particulièrement après l'année des libertés de 1990 au Cameroun, on a observé un changement d'attitude à l'égard de ce qui restait de l'idée du mouvement nationaliste au Cameroun. Le pouvoir a entrepris de décrisper la situation politique du pays en desserrant l'étau au niveau des libertés publiques, en introduisant le jeu démocratique et en réaménageant le multipartisme supprimé trente ans plus tôt. Ce contexte politique nouveau a donc été favorable au déclenchement d'une tentative de réhabilitation de la mémoire nationaliste et de sa formation politique, l'UPC, dans l'espace public national. Cependant, ce processus de réhabilitation amorcé va d'emblée souffrir de deux écueils dommageables : premièrement, il s'agit de la confusion créée et entretenue par les acteurs étatiques; deuxièmement, il s'agit de la confusion organisée par les héritiers putatifs et/ou autoproclamés de l'UPC.

\section{La coniusion créée et entretenue par les acteurs étatiques}

La loi du 16 décembre 1991 portant réhabilitation de certaines figures de l'histoire du Cameroun vise à réhabiliter certaines " grandes figures de l'histoire du Cameroun, aujourd'hui disparues, qui ont œuvré pour la naissance du sentiment national, l'indépendance ou la construction du pays, le rayonnement de son histoire ou de sa culture ». Dans cette formulation, elle vise nommément «MM. AhMadou Ahidjo, UM Nyobè Ruben, MoumiÉ Félix, Ouandí Ernest ».

De prime abord, on note qu'il y a certes un premier progrès dans la liste des personnes à réhabiliter. Tandis que les programmes d'histoire à l'école et au Lycée évoquaient juste Ruben UM NroBè, la loi de réhabilitation ajoute MouMIÉ Félix et OUANDIÉ Ernest. Toutefois, et malgré cette évolution dans l'énumération des figures historiques, ce texte suscite plusieurs réserves.

Tout d'abord, son intitulé prête à équivoque. II parle de « réhabilitation de certaines figures de l'histoire du Cameroun ". Cette formulation amène alors à se poser un certain nombre de questions : La liste de figures historiques énoncées est-elle indicative ou alors supplétive d'une précédente liste? Laisse-t-elle sous-entendre que d'autres figures ont certes «œuvré pour la naissance du sentiment national, l'indépendance ou la construction du pays ", mais que leur rôle n'a pas été assez éminent pour qu'elles soient considérées comme de "grandes figures de l'histoire du Cameroun », et donc qu'elles n'ont 
pas à être réhabilitées? Si l'on répond en considérant effectivement que la loi n'a voulu privilégier que les "grandes figures", alors on devrait expliquer en quoi les rôles de Ossendé AfANA, Abel KInguÉ, Pierre YeMBACK, Felix Roland MoumiÉ, Martin Singap, Ernest OuAndiÉ, Fotsing Raphael, TABeu Gabriel, Tankeu Noé, Marthe Moumie, MarieIrène NGAPETH BiYONG ou Ndeh NTUMAZAH, etc. furent moins éminents pour les faire figurer dans la liste des " grandes figures de l'histoire du Cameroun ». Si par contre on estime que par "certaines figures » il s'agissait de donner un caractère indicatif à l'énumération, alors l'État n'aura pas aidé à réhabiliter la mémoire nationaliste, mais tout simplement à la segmenter.

Ensuite, pourquoi parle-t-on de réhabilitation? Dans quoi et par rapport à quoi ces figures historiques sont-elles réhabilitées? Est-ce à dire qu'elles ont, par le passé, été déchues, diffamées, salies? Dans l'affirmative, que feraient alors côte à côte les noms de Ahmadou AHIDJO et Ruben UM NYobè, MoumiÉ Félix, Ernest OuANDIÉ ? De quelle déchéance, diffamation, salissure auraient-ils été victimes et qui justifieraient leur réhabilitation? Le texte ne le dit pas.

Enfin, c'est la présence dans la même liste d'Ahmadou AHIDJO et des leaders indépendantistes qu'il a combattus farouchement et dont il a contribué à bannir la mémoire et à criminaliser l'action qui peut choquer. Comment placer sur le même podium de l'histoire, mettre sur le même piédestal le bourreau et ses victimes? Si on considère que les nationalistes étaient effectivement des criminels ayant causé du tort à la nation, il n'est point besoin de les réhabiliter. II faudrait au contraire saluer l'action et rendre hommage à celui ayant contribué à leur chute, en l'occurrence l'ancien président Ahmadou AHIDJO. Si par contre, l'on admet que ces nationalistes faits " héros nationaux " ont été diffamés et ont vu leur mémoire salie, alors il faudrait les réhabiliter effectivement et souligner l'action dommageable de leur(s) oppresseur(s). En voulant faire une chose et son contraire, l'État contribue à obscurcir la mémoire nationale et à créer la confusion.

\section{La confusion orchestrée par les héritiers putatifis : l'instrumentation du "label UPC "}

Le retour au multipartisme aura paradoxalement été le déclencheur d'un processus de division par scissiparité au sein de l'UPC post-coloniale. En effet, dans sa configuration actuelle, I'UPC post-coloniale est le reflet d'un "schisme permanent ${ }^{47}$. Chaque faction reven- 
dique une légitimité historique. Chacune a ses militants, ses organes dirigeants, sa stratégie de positionnement, sa stratégie d'alliances électorales et gouvernementales etc. ${ }^{48}$ De fait, une des singularités parmi d'autres de l'UPC «[...] veut que l'aîné des partis camerounais ait passé trente quatre de ses quarante-trois années d'existence dans la clandestinité et que, depuis 1958, l'une des activités principales de I'UPC consiste à s'opposer à lui-même " ${ }^{49}$.

La confusion orchestrée et entretenue par les héritiers putatifs des nationalistes camerounais remonte beaucoup plus loin. Sa chronologie commence au moins en 1960 avec la création de "l'UPC légale " représentée par Mayi MATIP, Emma OTTU qui avaient abandonné le "maquis" pour se rallier au président Ahmadou AHIDJO. Mais cette "UPC légale " dotée d'un Bureau National Provisoire (BNP) va se voir absorber au sein de l'ancien parti unique l'Union Camerounaise (UC), devenue Union Nationale Camerounaise (UNC), puis RDPC en 1985. En 1962, suite au coup de force perpétré à Accra par WoUNGLY MASSAGA, NDO Michel et d'autres contre la branche de l'UPC exilée au Ghana, on assiste à la création de l'UPC-MANIDEM ${ }^{50}$ sous le nom de I'UPC du Comité révolutionnaire.

Avec l'annonce du retour au multipartisme, le processus de division- en perpétuelle évolution jusqu'aujourd'hui - commence en 1987. À cette date, l' "UPC originelle » représentée par Ndeh NTUMAZAH, s'associe avec d'autres groupes pour créer le Front Démocratique Camerounais (FDC). En 1990, ce sera au tour de "l'UPC du Prince Dika Akwa " ou "UPC du château » d'être créée avec le Prince DikA AKWA comme président et Augustin Frédéric KoDOCK comme secrétaire général, tous deux, anciens membres du parti unique et parti au pouvoir ${ }^{51}$.

Par la suite, diverses factions se développeront autour des leaders en compétition pour le prestige symbolique représenté par I'UPC. Ainsi on dénombrera, à côté de l'UPC -Ntumazah, l'UPC Dika, I'UPC-MANIDEM et l'UPC-Kodock, de nouvelles factions. C'est le cas

\footnotetext{
${ }_{48}^{8}$ Voy. Manga Olomo Joseph et NtUda Ebode Jean Vincent, Divisions au Coeur de l'UPC Contribution à la Connaissance de l'Histoire Politique du Cameroun, op. cit. p. 99.

${ }^{49}$ EyInga Abel, op. cit. p. 11.

50 Mouvement Africain pour la Nouvelle Indépendance et la Démocratie (MANIDEM).

${ }^{51}$ Voy. le récit fait par MANGa Olomo Joseph, NtUda EBode Jean Vincent, Divisions au Coeur de l'UPC Contribution à la Connaissance de l'Histoire Politique du Cameroun, op. cit. pp. 92 et suivantes; ALEGA MBELE Sévérin., 2002, "L'UPC s'empêtre dans les divisions », Cameroon-Info.Net, 17 avril 2002; MBIAPA Linda., "Cameroun - UPC : Vers la fin des divisions au sein des différentes factions », CAMERPOST, 16 février 2016; MBOuA Vincent, «Upc : Le schisme permanent », afrol News /Mutations, 11 janvier 2007 ; NDonkou Robert., "Cameroun: Henri Hogbè Nlend interdit à ANICET EKANÈ et Woungly MASSAGA de revendiquer leur appar- 
notamment de l'UPC-Mack-kit dite "UPC des fidèles », de l' "UPCHogbè " née à la suite d'une guerre de leadership entre Augustin Frédéric Kodock et Henri Hogbè NLEND. Selon l'un des leaders de faction upciste, «les tensions observées dans cette formation politique viennent du fait que chacun croit être le numéro un, le seul à même de succéder aux leaders historiques $\aleph^{52}$. Mais à cela, l'on pourrait ajouter que cette bataille autour du label UPC en cache deux autres: d'une part, la continuation d'un conflit latent que le gouvernement post-colonial n'a jamais cessé de mener contre l'idée incarnée par l'UPC dont la légitimité questionnerait la sienne; d'autre part la guerre de légitimité de la part d'acteurs politiques désireux de se positionner sur l'échiquier politique national, y compris en instrumentalisant le patrimoine historique et symbolique de l'UPC.

\section{Conclusion}

Au demeurant, la réhabilitation de la mémoire nationaliste au Cameroun reste d'actualité. Malgré la tentative d'effacement opérée sous le gouvernement d'Ahmadou AHIDJO, suivie d'une reconnaissance timorée et brumeuse sous le gouvernement de Paul BIYA, cette mémoire reste discrète mais vive dans la conscience de beaucoup de Camerounais. Au regard de l'enjeu que cette réhabilitation représente dans la mise en cohérence du récit national camerounais à (ré) écrire, et de sa réactualisation par la déclaration pourtant laconique du président français, il ne fait aucun doute que cette question demeure ouverte. Aussi, reste présent le risque de sa récupération et de son instrumentalisation politique par des acteurs en quête de légitimation.

\footnotetext{
${ }^{52}$ Ndonkou Robert, "Cameroun: Henri Hogbè Nlend interdit à ANICEt EkanÈ et Woungly Massaga de
} revendiquer leur appartenance à I'UPC », Cameroon-Info.Net, 26 avril 2015. 